\title{
A relação das práticas do Lean Manufacturing e o desempenho operacional: um estudo no setor de autopeças
}

Luis Henrique Rigato Vasconcellos

Doutor em Engenharia de Produção pela Escola Politécnica de São Paulo e Engenheiro de Produção pela Faculdade de Engenharia Industrial - FEI

Luis.vasconcellos@fgv.br

Fernando Coelho Martins Ferreira

Doutor e Mestre em Administração de Empresas pela Fundação Getulio Vargas - SP, Professor da Escola Superior de Propaganda e Marketing e da Fundação Getulio Vargas - SP. fernando.coelho@fgv.br

Marcos Souza dos Santos

Mestre Profissional em Gestão para Competitividade, Linha Supply Chain Management pela Fundação Getulio Vargas - SP, Engenheiro Mecânico pela Universidade Cruzeiro do Sul.

\section{Editor Científico: José Edson Lara}

Organização Comitê Científico

Double Blind Review pelo SEER/OJS

Recebido em 06.08.2019

Aprovado em 12.11.2019

Este trabalho foi licenciado com uma Licença Creative Commons - Atribuição - Não Comercial 3.0 Brasil 


\section{Resumo}

Título do Trabalho: A Relação das Práticas do Lean Manufacturing e o Desempenho Operacional: Um Estudo no Setor de Autopeças

Objetivo do estudo: investigar como a adoção das práticas de lean manufacturing se relaciona com o desempenho operacional dos processos organizacionais, medido a partir do indicador OEE (Overall Equipment Effectiveness).

Metodologia/abordagem: foi conduzido um estudo de caso em uma manufatura de filtros automotivos, utilizando uma abordagem mista quali-quantitativa. Os dados foram levantados através de um grupo focal com seis coordenadores de produção e da análise do levantamento histórico do indicador OEE no período de 2015 a 2017, na empresa estudada.

Originalidade/Relevância: o estudo fornece uma abordagem mais direta e imediata sobre a adoção prática do conceito de lean manufacturing no dia a dia de uma manufatura e sua relação com indicadores reais que mensuram o desempenho operacional de uma empresa.

Principais resultados: observou-se no estudo que os processos que possuem melhor desempenho operacional são necessariamente aqueles que possuem maior grau de aderência às práticas lean.

Contribuições teóricas/metodológicas: foram identificados indícios significativos que a adoções de práticas lean e o desempenho operacional estão diretamente relacionados. Tais resultados convergem com o referencial teórico levantado no estudo, que apontam que a adoção das práticas lean como estratégia de operações tem o potencial de eliminar uma série de desperdícios e, consequentemente, criar valor para a operação.

Contribuições para a gestão: a ferramenta desenvolvida no estudo permite medir o grau de aderência ao lean manufacturing, podendo ser útil para estudos futuros de análise e diagnóstico das práticas lean no meio empresarial.

Palavras-chave:Produção Enxuta; Lean Manufacturing; Práticas Lean; OEE; Desempenho Operacional.

\section{Abstract}

Title: The Relation of Lean Manufacturing Practices and Operational Performance: A Study in the Auto Parts Industry

Objective of the study: to investigate how the adoption of lean manufacturing practices relates to the operational performance of organizational processes, measured by the OEE (Overall Equipment Effectiveness) indicator. 
Methodology / approach: A case study was conducted in an automotive filter manufacture using a mixed qualitative and quantitative approach. Data were collected through a focus group with six production coordinators and the analysis of the historical data of the OEE indicator from 2015 to 2017 in the company studied.

Originality / Relevance: The study provides a more direct and immediate approach to the practical adoption of the concept of lean manufacturing its relationship to real indicators that measure a company's operating performance.

Main results: It was observed in the study that the processes that have better operational performance are necessarily those that have a higher degree of adherence to lean practices.

Theoretical / Methodological Contributions: Significant evidence has been identified that lean practice adoptions and operational performance are directly related. These results converge with the theoretical framework raised in the study, which shows that the adoption of lean practices as an operations strategy has the potential to eliminate a series of wastes and, consequently, create value for the operation.

Contributions to management area: the tool developed in the study allows measuring the degree of adherence to lean manufacturing and may be useful for future studies of analysis and diagnosis of lean practices in the business environment.

Keywords: Lean Production; Lean Manufacturing; Lean practices; OEE; Operational Performance.

\section{Resumen}

Título: La relación de las prácticas de manufactura lean y el desempeño operativo: un estudio en la industria de autopartes

Objetivo del estudio: investigar cómo la adopción de prácticas de manufactura lean se relaciona con el desempeño operativo de los procesos organizacionales, medidos desde el indicador OEE (OverallEquipmentEffectiveness).

Metodología / enfoque: se realizó un estudio de caso en una fabricación de filtros automotrices utilizando un enfoque cualitativo y cuantitativo mixto. Los datos fueron recolectados a través de un grupo focal con seis coordinadores de producción y el análisis de la encuesta histórica del indicador OEE de 2015 a 2017 en la compañía estudiada.

Originalidad / relevancia: el estudio proporciona un enfoque más directo e inmediato para la adopción práctica del concepto de manufactura lean en la manufactura cotidiana y su relación con indicadores reales que miden el desempeño operativo de una empresa.

Resultados principales: Se observó en el estudio que los procesos que tienen un mejor desempeño operativo son necesariamente aquellos que tienen un mayor grado de adherencia a las prácticas lean. 
Contribuciones teóricas / metodológicas: se ha identificado evidencia significativa de que las adopciones de prácticas lean y el desempeño operativo están directamente relacionados. Estos resultados convergen con el marco teórico planteado en el estudio, que muestra que la adopción de prácticas lean como estrategia de operaciones tiene el potencial de eliminar una serie de desechos y, en consecuencia, crear valor para la operación.

Contribuciones a la gestión: la herramienta desarrollada en el estudio permite medir el grado de adherencia a la manufactura lean, y puede ser útil para futuros estudios de análisis y diagnóstico de prácticas ajustadas en el entorno empresarial.

Palabras clave: Lean Production; Lean Manufacturing; Prácticas lean; OEE; Rendimiento operacional.

\section{1 - Introdução}

O conceito de lean manufacturing (traduzido para o português como manufatura enxuta) começou a ganhar notoriedade no meio acadêmico e empresarial a partir do fim dos anos 1980 e início dos anos 1990, especialmente após a publicação dos estudos de Womack, Jones e Roos (1992).O lean pode ser definido como uma forma de produzir bens e serviços de elevada qualidade e baixos custos, utilizando-se das vantagens da melhoria do fluxo produtivo por meio da eliminação dos desperdícios no fluxo de valor (LEE et al 2008).

Pesquisadores em gestão de operações dedicaram atenção considerável ao tema. Alguns trabalhos de destaque são os de Abdulmalek e Rajgopal (2007) utilizando uma simulação entre o "antes" e o "depois" da implementação do lean; Jabbour et al. (2013), através de um trabalho sofisticado de modelagem de equações estruturais relacionando o lean com o desempenho operacional ou,mais recentemente, Zhanet al. (2018), que realizaram um amplo estudo de práticas de manufatura enxuta em mais de 170 empresas chinesas.

A implementação da metodologia lean e de suas práticas, entretanto, pode carecer de uma abordagem mais direta e imediata. Questões ainda permanecem abertas quando o assunto é adotar o conceito no dia a dia da manufatura. As práticas lean realmente afetam o desempenho operacional, reduzindo, por exemplo, indicadores diretos de horas paradas e de refugo e retrabalho? Seria possível afirmar que diferentes setores dentro de uma mesma empresa que encaram diferentemente a questão sobre a filosofia lean apresentam resultados tão distintos? Afinal, vale a pena implantar o conceito lean e suas práticas?

Visando fazer frente a estes questionamentos, este trabalho tem como objetivo analisar a relação entre as práticas do lean manufacturing e o desempenho operacional dos 
processos que as adotam. Para o alcance deste propósito, estabeleceram-se como objetivos secundários deste estudo:(1) o levantamento da práticas de lean manufacturing que podem afetar o desempenho operacional dos processos de manufatura, medido por meio do indicador OEE (Overall Equipment Effectiveness); (2) a mensuração do grau de aderência às práticas lean em diferentes processos de manufatura; (3) a comparação entre os graus de aderência a essas práticas e o desempenho operacional dos processos investigados.

A verificação dessas questões pode prover importantes insights para o gestor de operações para esclarecer se a adoção de práticas lean pode afetar diretamente o desempenho de diferentes áreas; criar um instrumento que possa analisar a intensidade de implementação de práticas lean; contribuir para expansão do conceito de manufatura enxuta para melhoria de competitividade nas empresas. Neste trabalho, procura-se contribuir para esses aspectos, relatando quais práticas lean podem afetar o desempenho operacional de uma empresa e explorando a relação entre lean e desempenho.

Uma vez concluída a introdução deste trabalho, apresenta-se a revisão de literatura sobre as práticas de lean manufacturing e a medição do desempenho operacional por meio do indicador OEE. Em seguida, descrevem-se os procedimentos metodológicos do estudo de caso conduzido para, na sequência, discutir os resultados da pesquisa. Por fim, fazem-se as considerações finais do trabalho, expondo as suas contribuições e eventuais limitações.

\section{2 - Contexto da realidade investigada segundo a literatura}

No modelo lean manufacturing, o objetivo principal é criar valor para o cliente final, onde o fator custo tem papel importante. As organizações trabalham para não repassar aumento dos preços dos produtos para o cliente final, buscando alternativas para a redução de custos, o que possibilita aumento do seu lucro sem afetar o preço final do produto.

Conforme aponta Ohno (1997), a redução de custos deve ser o objetivo dos fabricantes de bens e consumo que busquem sobreviver no mercado. Durante um período de grande crescimento econômico, qualquer fabricante pode conseguir custos mais baixos com a produção maior, mas tal objetivo se torna mais difícil, e ainda mais importante, em momentos de retração econômica.

A filosofia lean manufacturing trabalha com o conceito de que, para redução de custos, é fundamental eliminar dos processos todas as atividades identificadas como desperdício. Womack e Jones (1998) afirmam que a eliminação do desperdício é importante para que todas 
as atividades ao longo de uma cadeia criem valor. A Toyota, por exemplo, identificou sete tipos principais de atividades que não agregam valor (superprodução, tempo de espera, transporte ou transferência, processamento incorreto, excesso de estoque, deslocamentos desnecessários e defeitos), afetando negativamente a produtividade da empresa, e incluiu um oitavo tipo de perda: a não utilização da criatividade dos funcionários (LIKER, 2007).

Conforme aponta Ohno (1997), a base do sistema de produção é a absoluta eliminação do desperdício, e sua sustentação se dá por meio do Just in Time e autonomação.

- Just in time significa que, em um fluxo, as partes corretas necessárias à montagem alcançam a linha de montagem no momento em que são necessárias e somente na quantidade necessária. Uma empresa que estabeleça esse fluxo pode chegar ao estoque zero. Para Dennis (2008) o objetivo do Just in Time é produzir um fluxo de valor contínuo para que o cliente possa puxar. É o instrumento certo para oferecer uma rápida resposta aos clientes, um melhor entendimento de tempo (takt) e de controle de anormalidades.

- Autonomação (jidoka) é conhecida como a automação com um toque humano. Em todas as fábricas da Toyota, a maioria das máquinas está equipada com seus dispositivos de segurança, parada de posição fixa, sistema de trabalho completo e sistemas pokayoke à prova de erros para impedir produtos defeituosos. Assim, um trabalhador pode atender diversas máquinas, tornando possível reduzir o número de operadores e aumentar a eficiência da linha. Quando ocorre um problema como parada de equipamento, isso força todos a tomarem conhecimento do fato.

\section{Overall EquipmentEffectiveness}

A mensuração do desempenho operacional de uma manufatura por meio do indicador OEE (Overall Equipment Effectiveness) é uma ferramenta de auxílio ao desenvolvimento de planos de ação para melhor desempenho dos equipamentos (IRFIRENE; BOUNIT; DAKKAK, 2017), baseando-se na identificação, medição e tomada de decisão para redução de perdas, sejam na forma de indisponibilidade do equipamento, sejam por meio de problemas de qualidade decorrentes do processo de fabricação (DAL; TUGNELL; GREATBANKS, 2000; LJUMBERG, 1998).

O OEE é calculado a partir de alguns componentes, descritos na tabela a seguir: 


\section{Tabela 1}

Descrição dos componentes do OEE

\begin{tabular}{|c|c|}
\hline Componente do OEE & Descrição \\
\hline $\begin{array}{l}\text { Disponibilidade do } \\
\text { Equipamento }\end{array}$ & $\begin{array}{l}\text { Mensura "quanto" o equipamento está disponível para ser utilizado pela produção. } \\
\text { Seu cálculo segue duas etapas: } \\
\text { I. A soma das horas de paradas planejadas (manutenção preventiva, } \\
\text { treinamentos, reuniões, etc.) e não planejadas do equipamento, subtraindo } \\
\text { as horas de refeições planejadas; } \\
\text { II. Divide-se o valor obtido da soma anterior pela quantidade de horas previstas } \\
\text { para trabalho do Equipamento. }\end{array}$ \\
\hline Eficiência & $\begin{array}{l}\text { Razão entre a quantidade de peças produzidas em um determinado período pela } \\
\text { quantidade de peças previstas para produção neste mesmo período. }\end{array}$ \\
\hline Qualidade & $\begin{array}{l}\text { Razão entre o número de peças produzidas e o número de peças aprovadas conforme } \\
\text { os critérios especificados pelo cliente. }\end{array}$ \\
\hline
\end{tabular}

Fonte: elaborado a partir de Ljumberg (1998) e Dal, Tugnell e Greatbanks (2000).

O OEE, expresso em valores percentuais, é calculado a partir do produto dos índices dos três componentes, conforme ilustra a fórmula a seguir:

$$
\begin{aligned}
& \text { Paradas não planejadas + } \\
& \text { OEE }=\frac{\text { Paradas planejadas }- \text { Refeições }}{\text { Horas de trabalho previstas }} \times \frac{\text { Peças Produzidas }}{\text { Peças Planejadas }} \times \frac{\text { Peças Aprovadas }}{\text { Peças Produzidas }}
\end{aligned}
$$

O ideal do OEE é tender a 100\%, indicando (a) máxima utilização do equipamento, (b) volume de produção integralmente dentro do plano e (c) todos os produtos atendendo aos requisitos de qualidade.

As práticas lean, se implantadas adequadamente, têm o potencial de eliminar desperdícios e agregar valor à empresa. A tabela 2 sintetiza as principais categorias de conceitos relacionados ao lean manufacturing, descrevendo seus efeitos potenciais sobre o OEE. Estas 14 categorias deram origem a um dos instrumentos de coleta de dados deste estudo.

\section{Tabela 2}

\begin{tabular}{|c|c|c|c|}
\hline Categoria & Efeito sobre o Indicador OEE & $\begin{array}{c}\text { Embasamento } \\
\text { Teórico }\end{array}$ & $\begin{array}{l}\text { Componente do } \\
\text { OEE Afetado }\end{array}$ \\
\hline Retrabalho & $\begin{array}{l}\text { O processo de retrabalho compromete a } \\
\text { disponibilidade porque significa ocupar a } \\
\text { carga máquina além do planejado. } \\
\text { Representa o processamento de um } \\
\text { produto mais vezes do que o previsto. }\end{array}$ & $\begin{array}{l}\text { Gama, Cavenaghi, } \\
(2009) \text {; } \text { Goodson } \\
(2002) .\end{array}$ & Disponibilidade \\
\hline $\begin{array}{l}\text { Setup forçado ou não } \\
\text { planejado }\end{array}$ & $\begin{array}{l}\text { O setup não planejado compromete a } \\
\text { disponibilidade porque representa a } \\
\text { paralisação da linha de produção para } \\
\text { troca de ferramental por mais tempo do } \\
\text { que o planejado. }\end{array}$ & $\begin{array}{l}\text { Falconi (2013); } \\
\text { Zhou (2016). }\end{array}$ & Disponibilidade \\
\hline
\end{tabular}

Categorias conceituais do Lean Manufacturing e seus efeitos sobre o OEE. 


\begin{tabular}{|c|c|c|c|}
\hline Categoria & Efeito sobre o Indicador OEE & $\begin{array}{c}\text { Embasamento } \\
\text { Teórico }\end{array}$ & $\begin{array}{l}\text { Componente do } \\
\text { OEE Afetado }\end{array}$ \\
\hline Excesso de produção & $\begin{array}{l}\text { A produção em excesso prejudica a } \\
\text { disponibilidade porque significa a } \\
\text { ocupação da linha de produção } \\
\text { processando um material não solicitado } \\
\text { pelo cliente, ocupando carga máquina e } \\
\text { capacidade de armazenamento de material } \\
\text { além do previsto. }\end{array}$ & $\begin{array}{l}\text { Nazareno, } \\
\text { Junqueira, Rentes } \\
(2004) .\end{array}$ & Disponibilidade \\
\hline Tempo de espera & $\begin{array}{l}\text { A linha parada por espera de material } \\
\text { prejudica a disponibilidade porque } \\
\text { representa a ociosidade de equipamento e } \\
\text { mão de obra, desperdiçando o potencial } \\
\text { produtivo da empresa. }\end{array}$ & $\begin{array}{l}\text { Ciarnienê, } \\
\text { Vienazindiene } \\
(2012)\end{array}$ & Disponibilidade \\
\hline $\begin{array}{l}\text { Ritmo ou cadência da } \\
\text { linha }\end{array}$ & $\begin{array}{l}\text { A falta de ritmo ou cadência da linha } \\
\text { representa o processamento de um } \\
\text { produto além do tempo previsto, } \\
\text { ocupando carga da máquina e capacidade } \\
\text { de produção acima do planejado. }\end{array}$ & Liker (2007) & Eficiência \\
\hline Just in Time \& Kanban & $\begin{array}{l}\text { O Just in Time praticado de maneira } \\
\text { incorreta prejudica a disponibilidade } \\
\text { porque produzir além da necessidade do } \\
\text { cliente final e possibilita o crescimento do } \\
\text { estoque. }\end{array}$ & $\begin{array}{l}\text { Rodrigues } \quad(2012) ; \\
\text { Dennis } \quad(2008) ; \\
\text { Rother (2010). }\end{array}$ & Disponibilidade \\
\hline Autonomação & $\begin{array}{l}\text { A autonomação impacta na qualidade, } \\
\text { pois, se desenvolvida em um grau de } \\
\text { pouca confiabilidade, pode comprometer } \\
\text { a qualidade do produto, gerando } \\
\text { retrabalhos e refugo. }\end{array}$ & $\begin{array}{lr}\text { Ohno } & \text { (1997); } \\
\text { Santos, } & \text { Gohr, } \\
\text { Gonçalves, } & \text { Vilar, } \\
\text { Arnaud (2009). }\end{array}$ & Qualidade \\
\hline Fluxo de Trabalho & $\begin{array}{l}\text { Um fluxo de trabalho com um grau de } \\
\text { baixa confiabilidade compromete a } \\
\text { disponibilidade pois pode acarretar } \\
\text { paradas de linha e grau baixo de } \\
\text { qualidade. }\end{array}$ & $\begin{array}{lr}\text { Ohno } & \text { (1997); } \\
\text { Santos, } & \text { Gohr, } \\
\text { Gonçalves, } & \text { Vilar, } \\
\text { Arnaud (2009). }\end{array}$ & Disponibilidade \\
\hline $\begin{array}{l}\text { Nivelamento de } \\
\text { Produção }\end{array}$ & $\begin{array}{l}\text { Um nivelamento de produção em um } \\
\text { estágio precário pode impactar a } \\
\text { disponibilidade, pois pode gerar excesso } \\
\text { de estoque, desperdício de recursos e } \\
\text { paradas das linhas de produção. }\end{array}$ & $\begin{array}{ll}\text { Rodrigues } & (2012) ; \\
\text { Dennis } \quad(2008) & \text {; } \\
\text { Rother (2010. }\end{array}$ & Disponibilidade \\
\hline $\begin{array}{l}\text { Equipes Multifuncionais } \\
\text { \& Operações } \\
\text { Padronizadas }\end{array}$ & $\begin{array}{l}\text { Uma prática de operações não } \\
\text { padronizadas ou equipes com baixo grau } \\
\text { de multifuncionalidade impactam tanto na } \\
\text { eficiência quanto na qualidade, devido a } \\
\text { procedimentos inadequados e critérios de } \\
\text { aceitação diferenciados. Ambas as } \\
\text { situações podem ocasionar tempo extra } \\
\text { para produção, retrabalhos ou refugo. }\end{array}$ & $\begin{array}{l}\text { Santos, } \quad \text { Gohr, } \\
\text { Gonçalves, Vilar, } \\
\text { Arnaud (2009). }\end{array}$ & $\begin{array}{l}\text { Eficiência; } \\
\text { Qualidade }\end{array}$ \\
\hline Excesso de Estoque & $\begin{array}{l}\text { O excesso de estoque prejudica a } \\
\text { disponibilidade, representando } \\
\text { armazenamento de material em processo } \\
\text { além do necessário, impactando na } \\
\text { continuidade do processo produtivo. }\end{array}$ & $\begin{array}{|lr|}\text { Ohno } & (1997) \\
\text { Dennis } & (2008) ; \\
\text { Santos, } & \text { Gohr, } \\
\text { Gonçalves, } & \text { Vilar, } \\
\text { Arnaud (2009). }\end{array}$ & Disponibilidade \\
\hline
\end{tabular}




\begin{tabular}{|c|c|c|c|}
\hline Categoria & Efeito sobre o Indicador OEE & $\begin{array}{c}\text { Embasamento } \\
\text { Teórico }\end{array}$ & $\begin{array}{l}\text { Componente do } \\
\text { OEE Afetado }\end{array}$ \\
\hline Gerenciamento Visual & $\begin{array}{l}\text { O Gerenciamento Visual tem potencial de } \\
\text { impactar os três componentes do OEE } \\
\text { (Disponibilidade, Eficiência } \\
\text { Qualidade). Isto ocorre porque ele permite } \\
\text { expor aos operadores informações dos } \\
\text { componentes do OEE, permitindo tomar } \\
\text { decisões mais precisas que auxiliem na } \\
\text { evolução dos componentes do OEE. }\end{array}$ & $\begin{array}{l}\text { Santos, } \quad \text { Gohr, } \\
\text { Gonçalves, Vilar, } \\
\text { Arnaud (2009). }\end{array}$ & $\begin{array}{l}\text { Disponibilidade; } \\
\text { Eficiência; } \\
\text { Qualidade }\end{array}$ \\
\hline $\begin{array}{l}\text { Controle de qualidade } \\
\text { zero defeito }\end{array}$ & $\begin{array}{l}\text { A falta de controle de qualidade é grave, } \\
\text { visto que o processamento de produtos de } \\
\text { qualidade duvidosa compromete a } \\
\text { confiabilidade do processo, gerando } \\
\text { possíveis retrabalhos ou refugo. }\end{array}$ & Zhou (2016) & Qualidade \\
\hline Manutenção Preventiva & $\begin{array}{l}\text { A manutenção preventiva realizada sem } \\
\text { planejamento adequado prejudica a } \\
\text { disponibilidade porque potencializa o } \\
\text { surgimento de paradas não planejadas } \\
\text { devido à quebra de equipamentos. }\end{array}$ & $\begin{array}{l}\text { Santos, Gohr, } \\
\text { Gonçalves, Vilar, } \\
\text { Arnaud (2009). }\end{array}$ & Disponibilidade \\
\hline
\end{tabular}

Fonte: elaborado pelos autores.

\section{3 - Diagnóstico da situação prática investigada}

O indicador OEE já era empregado na empresa onde este estudo foi desenvolvido. Tratase de uma indústria de filtros automotivos com uma área fabril de $25.000 \mathrm{~m}^{2} \mathrm{e}$ capacidade produtiva de aproximadamente 5.500.000 filtros/mês. Emprega cerca de 820 funcionários, dos quais 507 trabalham diretamente na linha de produção, que conta com aproximadamente 648 equipamentos. A figura 1 mostra o fluxo do processo produtivo, segmentado por células de produção denominadas “mini-fábricas” pela empresa.

O enfoque deste estudo recai sobre as "mini-fábricas" Elemento, Montagem e Pintura, em destaque na figura 1, visto que estas respondem pela maior parte do valor agregado durante o processo de produção e, conjuntamente, demandam mais de $70 \%$ de toda a mão de obra da manufatura. Cada célula de produção conta com gestores independentes e com autonomia para implementar práticas de gerenciamento distintas. 


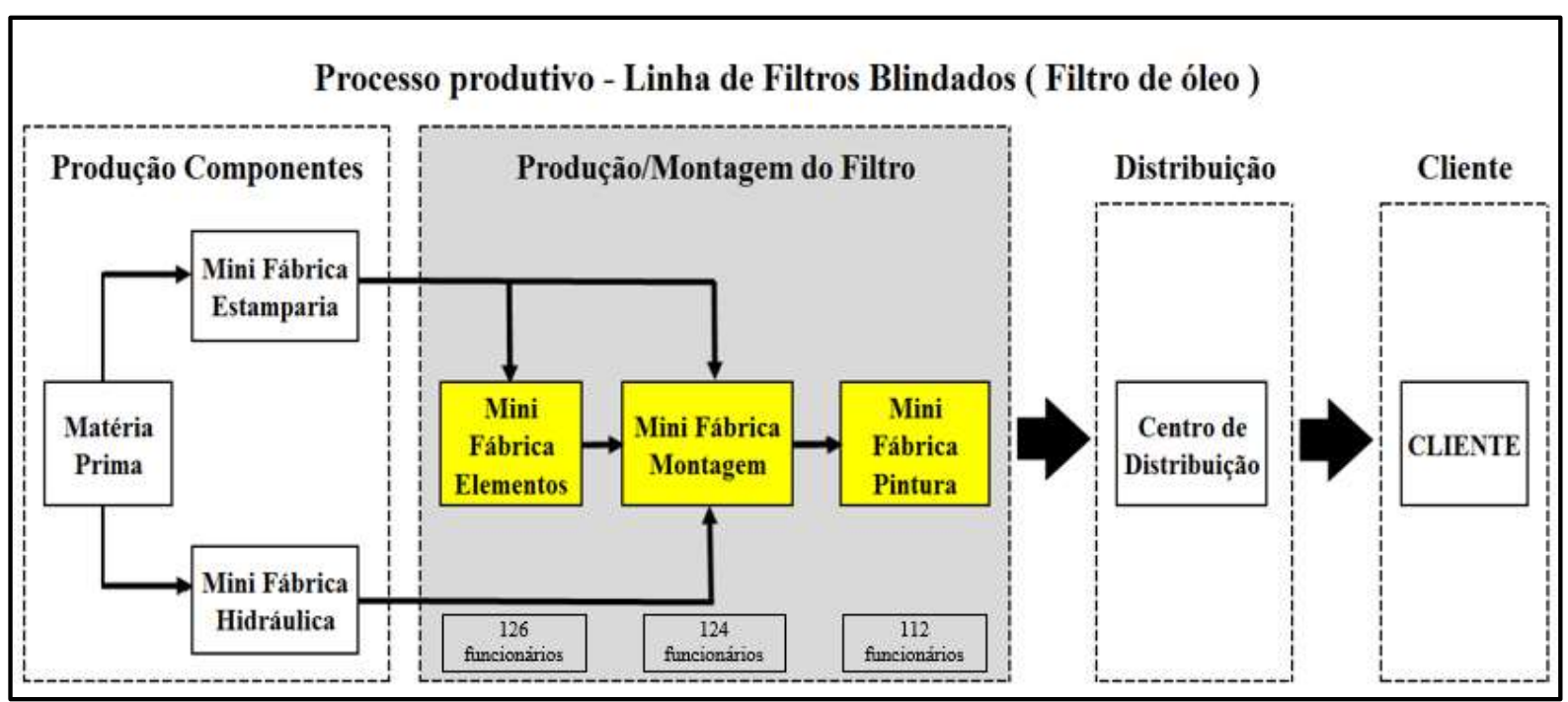

Figura 1 - Processo produtivo da linha de filtros blindados Fonte: elaborado pelos autores

Durante o período de 2015 a 2017, as três células em questão apresentaram índices de OEE distintos umas das outras, evidenciado uma variabilidade do desempenho entre áreas. Stefanovic (2014) afirma que, medindo e monitorando métricas contra objetivos predefinidos, as empresas podem fornecer valor agregado a grandes volumes de dados gerados ao longo do tempo. Este tipo de análise permite que as empresas rastreiem diversas métricas em diferentes níveis de organização e apresentem ações oportunas.

A tabela 1 apresenta o desempenho no indicador OEE das três células envolvidas no processo de Produção/Montagem do Filtro, nos dois turnos de trabalho, no período de 2015 a 2017, acompanhado de uma linha de tendência.Evidencia-se a notória variabilidade do desempenho operacional entre as três células de produção. Enquanto a célula Elemento apresenta um desempenho operacional muito superior (sempre acima de $70 \%$, chegando a picos de 95\%), com evidente tendência de melhoria, as outras duas têm índices OEE bem mais modestos, com tendência variando de estável (Montagem) a leve melhora (Pintura).

O desempenho operacional também pode ser verificado sob outras perspectivas. Nas três áreas analisadas, de acordo com informações fornecidas pela empresa, houve, neste mesmo período:

1. Desperdício de ativos, com equipamentos e mão de obra ociosos, totalizando um tempo de espera dos equipamentos de 3.580 horas;

2. Quase 4 milhões de filtros deixaram de ser produzidos;

3. Impacto no faturamento da empresa, com uma perda financeira estimada em $\mathrm{R} \$ 5.789 .764,00$. 
Uma vez reconhecidos esses impactos, investigar as razões de tamanha variabilidade de desempenho entre as células se torna uma questão primordial

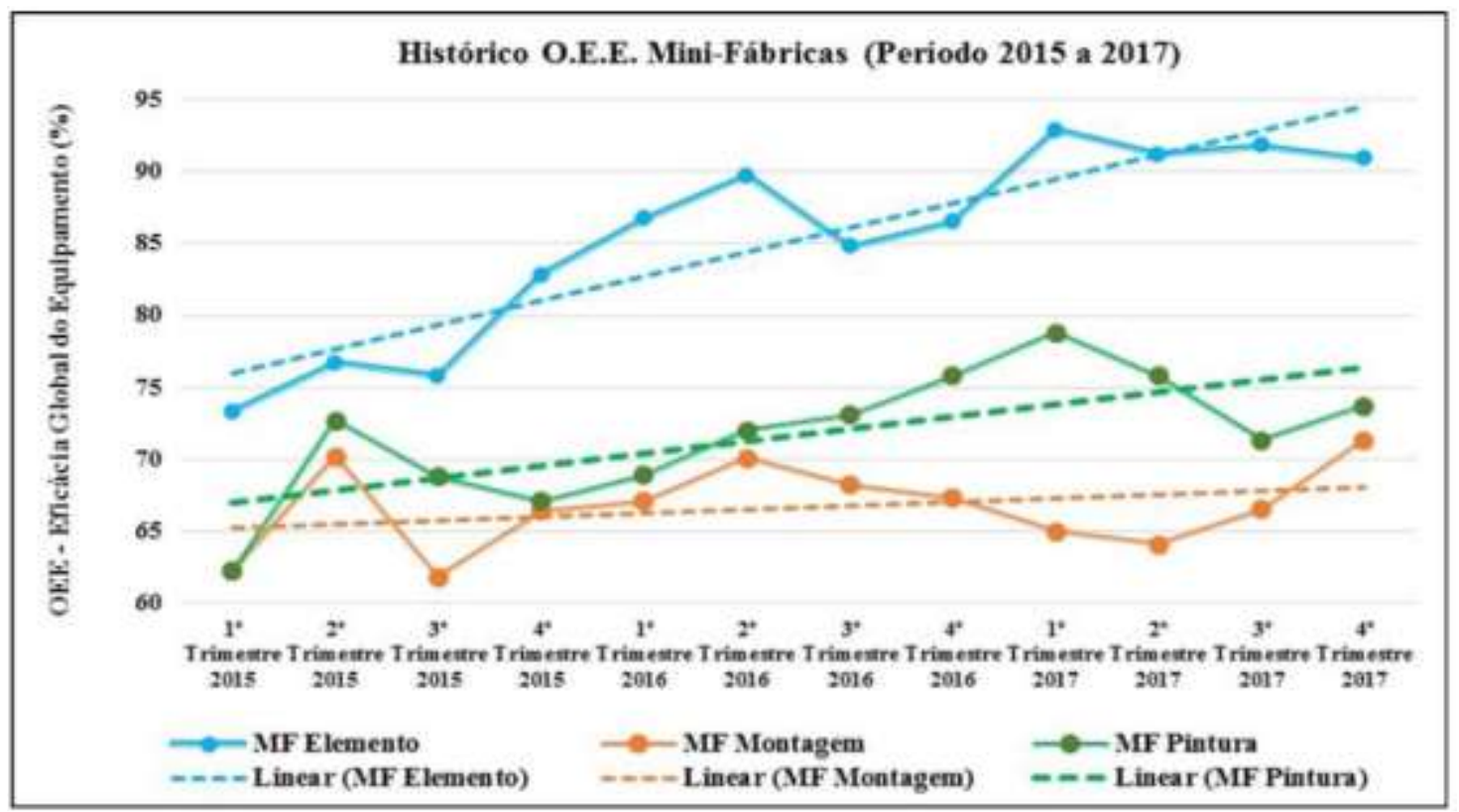

Gráfico 1 - Comportamento do OEE por célula de produção no período de 2015 a 2017 Fonte: elaborado pelos autores

Para Dennis (2008), o lean manufacturing se estrutura a partir da estabilidade e padronização dos processos, que fornecem as condições necessárias para a implantação do Just in Time e do jidoka. A meta do sistema, que só lograria sucesso por meio do envolvimento dos membros da equipe, deve ser sempre o foco no cliente.

\section{4 - Procedimentos metodológicos e formas de intervenção}

Como o objetivo do relato técnico é investigar como as práticas lean podem estar relacionadas com o desempenho operacional dos processos organizacionais, considerou-se apropriada uma abordagem quali-quantitativa. Conforme apontado por Ensslin e Vianna (2008), a discussão em relação ao projeto da pesquisa quali-quantitativa busca evidenciar que não há um modelo único para se construir conhecimentos confiáveis, e sim modelos adequados ou inadequados ao que se pretende investigar.

O estudo de caso é uma das mais importantes estratégias de investigação para fenômenos contemporâneos. Embora esse método esteja comumente associado a abordagens puramente qualitativas, ele pode também empregar um instrumento quantitativo (DOONLEY, 2002; DENZIN; LINCOLN, 1994). 
Yin (2015) aponta basicamente três fatores para definição do método de pesquisa: a) A forma da questão de pesquisa; b) $\mathrm{O}$ fato de o pesquisador ter pouco ou nenhum controle dos eventos comportamentais; c) o foco de estudo ser um fenômeno contemporâneo (em vez de um fenômeno completamente histórico).

Conforme aponta Yin (2015), estudo de caso é uma investigação empírica que apura um fenômeno contemporâneo (o "caso") em profundidade e em seu contexto de mundo real, especialmente quando os limites entre fenômeno e o contexto puderem não ser claramente evidentes. Complementa Severino (2016) que o estudo de caso se concentra em um caso particular, considerado representativo de um conjunto de casos análogos, por ele significativamente representativo.

O estudo de caso deste relato técnico apresentou as características pertinentes ao método de pesquisa citadas por Creswell (2010) e Yin (2015):a investigação foi desenvolvida em uma indústria de autopeças, onde os participantes vivenciam o problema sob investigação. O próprio pesquisador coleta os dados utilizando mais de uma fonte (plataformas digitais $\mathrm{e}$ entrevistas).Para avaliação do desempenho local, o pesquisador utiliza o conceito da própria organização que, é a segmentação de um processo macro em células de produção denominadas mini-fábricas.A interpretação final da investigação do problema e as possíveis causas são baseadas no cruzamento das informações adquiridas no processo de coleta de dados, utilizando plataformas digitais da empresa e entrevistas realizadas pelos participantes do processo, que serão detalhados a seguir.

Adotaram-se dois procedimentos de coleta para a pesquisa quantitativa. $\mathrm{O}$ primeiro foi a obtenção de dados por meio das plataformas digitais da empresa (GTR - Gerenciamento em Tempo Real), referente ao desempenho do OEE das trêscélulas de produção,no período de 2015 a 2017. Uma segunda plataforma foi consultada para o controle de refugo, visando estimar o custo dos filtros não entregues ao cliente final.

O segundo procedimento foi a aplicação de um instrumento quantitativo dentro de um grupo focal. A formação de um grupo focal consiste na reunião de um grupo de especialistas, para que o pesquisador possa coletar dados, visando entender como estes especialistas pensam a respeito de um determinado fenômeno (KRUEGER; CASEY, 2000). As discussões são conduzidas por um moderador, que pode ser o pesquisador ou um entrevistador independente, desde que esteja previamente preparado e munido de uma lista de perguntas e tópicos a serem discutidos (MUNARETTO; CORRÊA; ARAÚJO, 2013). 
Para compor o grupo focal foram selecionados intencionalmente os coordenadores de Produção. Conforme descreve Creswell (2010), é comum em estudos de caso ocorrer a seleção intencional dos participantes ou dos locais (ou dos documentos ou do material visual) que melhor ajudarão o pesquisador a entender os problemas e a questão de pesquisa, o que não sugere, necessariamente, uma amostragem ou seleção aleatória de um grande número de participantes e locais.Os motivos para a escolha intencional deste público-alvoforam:

- A função dos Coordenadores de Produção na gestão das trêscélulas sob análise, onde seu limite de atuação se encontra entre a Supervisão de Produção e os Operadores de Produção;

- Os Coordenadores de Produção atuam diretamente na operação envolvendo os dois turnos de trabalho da fábrica;

- Evitar respostas tendenciosas dos responsáveis diretos de cada célula de produção (Supervisores de Produção).

A tabela 3 apresentaum breve perfil dos participantes do grupo focal.

\section{Tabela 3}

Perfil dos participantes do grupo focal

\begin{tabular}{|c|c|c|c|}
\hline Participante & Formação Acadêmica & Tempo de Experiência na Função & Turno de Trabalho \\
\hline 1 & Tecnólogo em Logística & 9 anos e 8 meses & $1^{\mathbf{o}}$ \\
\hline 2 & Engenheiro Mecânico & 7 anos e 8 meses & $1^{\mathbf{o}}$ \\
\hline 3 & Engenheiro Mecânico & 7 anos e 8 meses & $1^{\mathbf{o}}$ \\
\hline 4 & Processos Gerenciais & 6 anos e 1 mês & $2^{\mathbf{o}}$ \\
\hline 5 & Tecnólogo em Logística & 1 anos e 8 meses & $2^{\mathbf{o}}$ \\
\hline 6 & Tecnólogo em Logística & 1 anos e 8 meses & $2^{\mathbf{o}}$ \\
\hline
\end{tabular}

Fonte: elaborado pelos autores

Para a coleta de dados foi elaborado, primeiramente, um questionário-piloto. Para avaliação e validação deste questionário, foram realizadas duas entrevistas preliminares com dois Coordenadores de Produção, com o objetivo de a) avaliara adequação da linguagem utilizada noinstrumento de coleta; b) ajustar a estrutura das perguntas para facilitar o grau de entendimento;c) verificar a objetividade das perguntas no que se refere à obtenção das informações solicitadas.

Após a aplicação do questionário-piloto foram realizados ajustes para ampliação de sua aplicação aos demais entrevistados no grupo focal, visando levantar as informações com os coordenadores de maneira simultânea, sem possíveis vieses de respostas. Para isso, os entrevistados foram dispostos em um auditório de forma que não interagissem durante o 
preenchimento do questionário. Podia-se interagir somente com o pesquisador, em caso de eventuais dúvidas.

Durante o grupo focal, o mediador projetava a perguntas aos coordenadores que tinham o instrumento de coleta impressos. Todos os coordenadores de produção respondiam as perguntas simultaneamente, seguindo os princípios do método Delphi sugeridos por Stebbins (2008). A aplicação foi realizada no auditório da própria empresa no estudo de caso durante o mês de junho de 2018 .

O instrumento desenvolvido e aplicado no grupo focal avaliou a intensidade da utilização das práticas do lean manufacturing em cada célula, conforme escala de Likert em cinco níveis de intensidade, a saber: 1 - A prática nunca é utilizada; 2 - A prática raramente é utilizada;3 - A prática é utilizada algumas vezes;4 - A prática é utilizada muitas vezes;5 - A prática sempre é utilizada.

O instrumento de coleta para o grupo focal foi composto por 14 categorias que representaram as práticas do lean manufacturing, conforme referencial teóricopreviamente apresentado na tabela 2. Cada categoria continha duas questões, totalizando, portanto, 28perguntas.

\section{5 - Contribuições técnológicas: a análise dos resultados}

As tabelas a seguir discutem o grau de aderência de cada uma das células às práticas do lean manufacturing, estimado a partir do grupo focal conduzido in loco.O grau de aderência às práticas, organizadas a partir das 14 categorias apresentadas no tabela 4, é medido por meio de um score que pode variar de 1 a 25 pontos. Esta pontuação resulta do produto entre as duas questões que medem a intensidade de utilização das práticas lean de cada categoria. Dessa maneira, por exemplo, se em determinada categoria as respostas dos participantes foram $5 \mathrm{em}$ cada uma das duas questões que a compõe, o score será 25. Se, hipoteticamente, determinada célula tivesse um grau de aderência de $100 \%$ a todas as categorias, a soma de todos os scores daria $350(14 \times 25)$.

Os primeiros resultados podem ser discutidos a partir da tabela 4, que apresenta os scores de aderência a cada prática do lean manufacturing por célula de produção e por turno. 


\section{Tabela 4}

Grau de aderência às práticas do lean manufacturing(por turno de trabalho)

\begin{tabular}{|c|c|c|c|c|c|c|}
\hline \multirow{2}{*}{ Categoria } & \multicolumn{3}{|c|}{$1^{\circ}$ Turno } & \multicolumn{3}{|c|}{$2^{\circ}$ Turno } \\
\hline & Elemento & Montagem & Pintura & Elemento & Montagem & Pintura \\
\hline Retrabalho & 16 & 9 & 8 & 16 & 4 & 6 \\
\hline Setup forçado ou não planejado & 20 & 12 & 12 & 12 & 12 & 20 \\
\hline Excesso de produção & 1 & 20 & 20 & 1 & 25 & 20 \\
\hline Tempo de espera & 15 & 6 & 6 & 16 & 9 & 12 \\
\hline Ritmo ou cadência da linha & 12 & 9 & 6 & 15 & 2 & 6 \\
\hline Just in Time\& Kanban & 20 & 16 & 12 & 20 & 9 & 9 \\
\hline Autonomação & 9 & 16 & 4 & 25 & 8 & 4 \\
\hline Fluxo de Trabalho & 25 & 6 & 6 & 8 & 2 & 12 \\
\hline Nivelamento de Produção & 20 & 9 & 9 & 20 & 2 & 12 \\
\hline $\begin{array}{l}\text { Equipes Multifuncionais \& } \\
\text { Operações Padronizadas }\end{array}$ & 6 & 6 & 2 & 12 & 4 & 6 \\
\hline Excesso de Estoque & 20 & 4 & 2 & 25 & 1 & 12 \\
\hline Gerenciamento Visual & 12 & 9 & 4 & 10 & 4 & 12 \\
\hline $\begin{array}{l}\text { Controle de qualidade zero } \\
\text { defeito }\end{array}$ & 25 & 12 & 15 & 25 & 4 & 12 \\
\hline Manutenção Preventiva & 16 & 6 & 8 & 16 & 1 & 9 \\
\hline Score Total & 217 & 140 & 114 & 221 & 87 & 152 \\
\hline Percentual de Aderência & $62,00 \%$ & $40,00 \%$ & $32,57 \%$ & $63,14 \%$ & $24,86 \%$ & \\
\hline
\end{tabular}

Fonte: elaborado pelos autores

Os dados dispostos na tabela demonstram que a célula de produção Elemento possui um grau de aderência às práticas do lean manufacturing sensivelmente superior ao das demais células. Elemento, Montagem e Pintura apresentam graus bastante distintos de aderência às práticas lean.Tal fato pode ser mais bem observado nas linhas que apresentam a soma total dos scores e o percentual de aderência às práticas, estimado a partir da razão entre o score total de cada célula e o score máximo possível (350 pontos).

Mais instigante, entretanto, é a variabilidade na percepção do grau de aderência entre turnos. Enquanto a célula Elemento quase não apresenta diferença no grau de aderência entre o $1^{\circ}$ e o $2^{\circ}$ turno, as outras duas células apresentam considerável variabilidade na comparação entre esses períodos de trabalho. No caso da célula Montagem, o mais grave, essa diferença supera os 15 pontos percentuais. Apesar de não terem sido investigadas, as causas para tamanha variabilidade entre turnos podem estar relacionadas a diversos fatores, desde aspectos motivacionais e comportamentais até questões relacionadas a gestão e estilos de liderança, dentre muitos outros. 
Complementando a tabela anterior, a tabela 5 elimina a discriminação por turnos e expõe a pontuação média de cada categoria para as três células de produção. Esses scores médios, mais uma vez, demonstram a superioridade no grau de aderência da célula Elemento, que apresenta as melhores pontuações (em destaque) em todas as categorias, exceto naquela relacionada ao excesso de produção, um desperdício inerente à maioria dos processos produtivos empurrados.

Evidentemente, esses scores médios também permitem identificar, por célula e no conjunto,as práticas lean que possuem menor aderência no processo de produção e montagem do filtro, demandando eventuais ajustes da gerência da empresa. Nesse caso, as práticas relacionadas às equipes multifuncionais e operações padronizadas, ritmo e cadência de linha e gerenciamento visual são aquelas que inspiram maiores cuidados, conforme revela a pontuação média de cada categoria.

\section{Tabela 5}

Grau de aderência às práticas do lean manufacturing (consolidado)

\begin{tabular}{|l|c|c|c|c|}
\hline \multicolumn{1}{|c|}{ Categoria } & Elemento & Montagem & Pintura & $\begin{array}{c}\text { Médiada } \\
\text { Categoria }\end{array}$ \\
\hline Retrabalho & 16 & 6,5 & 7 & 9,83 \\
\hline Setup forçado ou não planejado & 16 & 12 & 16 & 14,67 \\
\hline Excesso de produção & 1 & 22,5 & 20 & 14,50 \\
\hline Tempo de espera & 15,5 & 7,5 & 9 & 10,67 \\
\hline Ritmo ou cadência da linha & 13,5 & 5,5 & 6 & 8,33 \\
\hline Just in Time\& Kanban & 20 & 12,5 & 10,5 & 14,33 \\
\hline Autonomação & 17 & 12 & 4 & 11,00 \\
\hline Fluxo de Trabalho & 16,5 & 4 & 9 & 9,83 \\
\hline Nivelamento de Produção & 20 & 5,5 & 10,5 & 12,00 \\
\hline Equipes Multifuncionais \& Operações Padronizadas & 9 & 5 & 4 & 6,00 \\
\hline Excesso de Estoque & 22,5 & 2,5 & 7 & 10,67 \\
\hline Gerenciamento Visual & 11 & 6,5 & 8 & 8,50 \\
\hline Controle de qualidade zero defeito & 25 & 8 & 13,5 & 15,50 \\
\hline Manutenção Preventiva & 16 & 3,5 & 8,5 & 9,33 \\
\hline Score Total & $\mathbf{2 1 9}$ & $\mathbf{1 1 4}$ & $\mathbf{1 3 3}$ & 155,33 \\
\hline Percentual de Aderência & $62,57 \%$ & $32,43 \%$ & $38,00 \%$ & $44,33 \%$ \\
\hline Font ena & & & 7 \\
\hline
\end{tabular}

Fonte: elaborado pelos autores

Os valores consolidados por célula de produção,quando confrontados com os dados a respeito do desempenho no indicador OEE referentes ao período de 2015 a 2017, pretendem demonstrar como esse indicador, tomado como proxy para o desempenho operacional, e o grau 
de aderência às práticas lean estão relacionados. A tabela 6 organiza essas informações por célula de produção.

\section{Tabela 6}

Relacionamento entre as práticas do lean manufacturing e o indicador OEE.

\begin{tabular}{|c|c|c|c|c|c|c|}
\hline $\begin{array}{c}\text { Célula de } \\
\text { Produção }\end{array}$ & $\begin{array}{c}\text { Score } \\
\text { Total }\end{array}$ & $\begin{array}{c}\text { Grau de } \\
\text { Aderência (\%) }\end{array}$ & OEE (\%) & $\begin{array}{c}\text { Tempo Ocioso } \\
\text { (Horas) }\end{array}$ & $\begin{array}{c}\text { Estimativa } \\
\text { de filtros não } \\
\text { produzidos }\end{array}$ & Perda estimada \\
\hline Elemento & 219 & 62,57 & 84,55 & 177 & 254.100 & $\mathrm{R} \$ 315.050,00$ \\
\hline Montagem & 114 & 32,43 & 71,95 & 828 & 1.170 .991 & $\mathrm{R} \$ 1.681 .600,00$ \\
\hline Pintura & 133 & 38,00 & 64,80 & 2.575 & 2.514 .387 & $\mathrm{R} \$ 3.793 .114,00$ \\
\hline
\end{tabular}

Fonte: elaborado pelos autores.

Os dados da tabela 4 apontam que a célula de produção que possui o melhor desempenho no OEE é justamente a que também possui o maior grau de aderência ao lean. Com um índice de 84,55\% de OEE, a célula Elemento se distancia das células de Montagem e Pintura, que apresentaram indicadores bem mais modestos e com pequena diferença uma da outra. Essas duas células também apresentaram pequena diferença quanto à aderência às práticas lean. Tais resultados sugerem que um desempenho operacional superior está diretamente relacionado à adoção das práticas do lean manufacturing, tomadas a partir das 14 categorias discutidas neste trabalho.

Dado que a manufatura "enxuta" mira alta eficiência em seus processos, resultando em elevada utilização dos recursos e custos operacionais menores, os dados que complementam a tabela 4, obtidos diretamente com a empresa, dão dimensão do custo que se paga pela ineficiência. Conforme previamente discutido, do período de 2015 a 2017 as três células de produção ficaram paradas durante 3.580 horas, o que representou a não produção de quase 4 milhões de filtros e uma receita não realizada de quase seis milhões de reais. Entretanto, conforme discrimina a tabela, a participação da célula Elemento no custo dessa ineficiência é mínimo. As horas paradas desta célula respondem por menos de 5\% do total, representando cerca de $6,5 \%$ dos filtros que deixaram de ser produzidos e aproximadamente 5,5\% da receita não realizada.Montagem e Pintura, aquelas com menor grau de adesão às práticas lean e menor OEE, respondem pelo restante, o quê, em termos práticos, se traduz em enormes perdas financeiras à organização. 


\section{6 - Considerações finais}

Este trabalho teve como objetivo compreender a relação entre as práticas do lean manufacturing e o desempenho operacional, tendo como objeto de pesquisa uma empresa fabricante de filtros para o setor automotivo. Por meio de uma abordagem quali-quantitativa, marcada pela análise de dados sobre o indicador OEE no período de 2015 a 2017 e pela condução de um grupo focal com participantes que ocupam posições-chave nas células de produção pesquisadas, foram identificados indícios que a adoções de práticas lean e o desempenho operacional estão diretamente relacionados. Tais resultados convergem com o referencial teórico levantado, que apontam que a adoção das práticas lean como estratégia de operações tem o potencial de eliminar uma série de desperdícios e, consequentemente, criar valor para a operação.

Pôde-se observar, também, a considerável variabilidade no indicador OEE dentro da própria organização, quando se consideram as três células de produção selecionadas para o estudo, aparentemente em função dos diferentes graus de aderência às práticas lean que estas possuem. Além disso, as áreas de produção com menor aderência, além de se desempenharem pior no OEE, também apresentam uma considerável variabilidade nesse indicador,quando se consideram os dois turnos de produção da empresa analisada.Tais comportamentos convergem comum dos efeitos desejados com a adoção das práticas lean: a estruturação de processos que, além de entregarem maior conformidade (atendimento aos requisitos do cliente), gerem maior consistência (menor variabilidade).

Do ponto de vista gerencial, a ferramenta estruturada para medir o grau de aderência ao lean manufacturing permitiu que a empresa estudada identificasse tanto as práticas que estão mais incorporadas à rotina da manufatura quanto aquelas que ainda estão longe do ideal. Além de sua evidente aplicabilidade em estudos acadêmicos, esta ferramenta, portanto, também se mostrou útil para análise e diagnóstico das práticas lean no meio empresarial.

É importante frisar, entretanto, que a delimitação metodológica do trabalho impede que se estabeleça uma relação de causalidade entre as práticas lean e o desempenho operacional. Em outras palavras, pode-se dizer que essas duas variáveis estão relacionadas, mas não necessariamente que uma cause a outra. Isso demandaria alterações no desenho de pesquisa que, além de buscarem identificar tal causalidade, também eliminassem a possibilidade de uma correlação espúria entre essas variáveis.

O caráter eminentemente exploratório deste estudo, ao mesmo tempo em que impede a generalização de seus resultados, abre oportunidades para que entre as práticas lean e o 
desempenho operacional sejam mais bem compreendidas em trabalhos futuros. Nesse sentido, (i) a ampliação do universo amostral, englobando uma quantidade relevante de empresas dos mais variados setores, (ii) a observação de todas as etapas de um processo de manufatura, ao invés de somenteas etapas consideradas mais relevantes, e (iii)a incorporação de outros indicadores para compor o construto desempenho operacional poderiam trazer importantes contribuições para a discussão dessa relação.

\section{Referências}

ABDULMALEK, F. A.; RAJGOPAL, J.Analyzing the benefits lean manufacturing and value stream mapping via simulation: a process sector case study. International Journal of Production Economics, v.107, n.1, p.223- 236, 2007.

CIARNIENÊ, Ramunê; VIENAZINDIENE, Milita. Lean Manufacturing: Theory and Practice.Economicsand Management: 2012.

CRESWELL, John W. Projeto de Pesquisa: Métodos Qualitativo, Quantitativo e Misto. $3^{\mathrm{a}}$ edição. Porto Alegre: Artmed, 2010.

DAL, Bulent; TUGWELL, Phil; GREATBANKS, Richard. Overall equipment effectiveness as a measure of operational improvement - A practical analysis.International Journal of Operations \& Production Management, Vol. 20 Issue: 12, pp.1488-1502, 2000.

DENNIS, P. ProduçãoLeanSimplificada. $2^{a}$ edição. Porto Alegre: Bookman, 2008.

DENZIN, N.K., Lincoln, Y.S., (1994). Handbook of qualitative research. Thousand Oaks (CA): Sage Publications.

ENSSLIN, Leonardo; VIANNA, William Barbosa. O Design na pesquisa quali-quantitativa em engenharia de produção- questões epistemológicas. Revista Produção Online, v8, n.1, 2008.

GAMA, Kleber T.; CAVENAGHI, Vagner. Medidas de Desempenho e Produção Enxuta: fundamentos e propostas para um sistema de medição do desempenho. XXIX ENCONTRO NACIONAL DE ENGENHARIA DE PRODUÇÃO, Salvador. Anais. Salvador: 2009.

GOODSON, R. E. Read a Plant-Fast. Harvard Business Review. Vol. 80, p105-113. 2 maio2002.

JABBOUR, A. B. L. D. S., JABBOUR, C. J. C., Freitas, W. R. D. S., \& Teixeira, A. A. Lean and green? Empirical evidence from the Brazilian automotive industry. Gestão\&Produção, 20(3), 653-665, 2013.

KRUEGER, R. A.; CASEY, M. A. Focus groups. A practical guide for applied research. 3. ed. [S.1.]: Thousand Oaks, 2000

LEE Sang M;OLSON David L.; LEE Sang-Heui; HWANG Taewon; SHIN Matt S.; Entrepreneurial applications of the lean approach to services industries.The Service Industries Journal. Vol.28, nº7 , p973-987. Sep. 2008.

LIKER, J. K. O modelo Toyota: manual de aplicação. Porto Alegre: Bookman, 2007.

MUNARETTO, L. F.; CORRÊA, L. H.; ARAÚJO, J. C. D. C. Um estudo sobre as características do método Delphi e de grupo focal, como técnicas na obtenção de dados em pesquisa exploratória. Revista de Administração da Universidade Federal de Santa Maria, Santa Maria, v. 6, n. 1, p. 9-24, março 2013.

NAZARENO, Ricardo R.; JUNQUEIRA, Roberta P.; RENTES, Antônio F.O impacto do Sistema Lean de Desenvolvimento na estrutura organizacional da área de engenharia: um estudo de caso. XI SIMPEP, 2004, Bauru. Anais. Bauru: 2004. 
OHNO, T.O sistema Toyota de produção - além da produção em larga escala. Porto Alegre: Bookman, 1997.

RODRIGUES, M. Implementação de práticas Lean numa linha de produção eletrônica. Faculdade de Engenharia da Universidade do Porto, Jul-2012.

ROTHER, M. Toyota Kata: gerenciando pessoas para melhoria, adaptabilidade e resultados excepcionais. Porto Alegre: Bookman, 2010.

SEVERINO, Antônio J. Metodologia do trabalho científico. 24 edição, São Paulo: Cortez, 2016.

STEBBINS, R. A. Exploratory research in the social sciences.Qualitative research method.Thousand Oaks, Sage, 2001.

STEFANOVIC N.Proactive Supply Chain Performance Management with Predictive Analytics.TheScientificWorldJournal, pp. 528917, out. 2014.

WOMACK, J.P., JONES, D.T. \& ROSS, D. The Machine that Changed the World.Rawson Associates, New York, NY, 1992.

WOMACK, J. P., JONES, D. T. A Mentalidade Enxuta nas Empresas: elimine o desperdício e crie riqueza. Rio de Janeiro: Campus, 1998.

YIN, Robert K. Estudo de caso: planejamento e métodos. $5^{\text {a }}$ edição. Porto Alegre: Bookman, 2015.

ZHAN, Y.; TAN, K.T; JI, G; CHUNG, L. CHIU, A.S.F. Green and lean sustainable development path in China: Guanxi, practices and performance. Resources, Conservation and Recycling. Volume 128, January 2018, Pages 240-249

ZHOU, Bin. Lean principles, practices, and impacts: a study on small and medium-sized enterprises (SMEs). Annals of Operations Research, Vol. 241, p.457-474, Jun.2016. 